kontrolle über einen Krypto-Chip mit Benutzerauthentifizierung über PIN-Code sind nur einige Beispiele für den Einsatz der patentrechtlich geschützten certgate Technologie.

Mit certgate Technologie geschützte NFC-fähige Smartphones und Tablets können konventionelle Kredit-, ec- oder Debit-Karten, Zertifikate für die Anmeldung an PC oder Netzwerk, Fahrscheine für öffentliche Verkehrsmittel oder Mitarbeiterausweise beinhalten und mit Eingabe des richtigen PIN-Codes die erforderliche digitale Authentisierung auslösen.

Der Einsatz von certgate Sicherheitstechnologien ermöglicht damit den mobilen Einsatz der echten Zwei-Faktor-Authentisierung, also der Kombination aus Wissen und Besitz - beginnend beim USB-Stick über Bluetooth(tm) und IP Cloud bis hin zu modernen NFC-Anwendungen. Insbesondere die NFC-Technologie wird als allgegenwärtige Infrastruktur in sehr kurzer Zukunft den Alltag in unserer Gesellschaft erobert haben.

Zum Bereich der mobilen Endgeräte, die certgate Technologie unterstützen, gehören aktuell Betriebssysteme wie Android, BlackBerry(tm) und Windows.

Laufend kommen neue Plattformen hinzu, die als Host-Systeme den Komfort und die Sicherheit von microSD(tm)-Speicherkarten unterstützen. Die certgate Sicherheitsanwendungen nutzen dieses Format mit einem zusätzlich integrierten On-Card-Smartcard-Prozessor der neuesten Generation in einem treiberlosen Betrieb, also ohne Änderungen der SD-Bus-Treibersoftware des Smartphones. Diese einfache Integration in die Geräte ist der Hauptgrund dafür, dass die ausgereifte und bewährte certgate Technologie von Partnern in aller Welt zur Härtung einer Vielzahl von Security-Lösungen eingesetzt wird. PKI-Unterstützung, Workstation-Anmeldung mit Zertifikat und Sprachverschlüsselung gehören zu den von Partnern realisierten, leistungsfähigen Lösungen für Smartphones, Tablets und Notebooks.

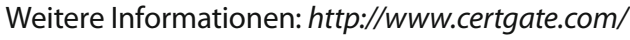

\section{IT.NRW: Elektronische Übermittlung von Elterngeldbescheinigungen an die Finanzverwaltung}

Der Landesbetrieb Information und Technik Nordrhein-Westfalen (IT.NRW) hat für NRW flächendeckend die elektronische Übermittlung von Leistungsbescheinigungen der Elterngeldempfänger/-innen an die Finanzverwaltung umgesetzt.

Laut Vorgabe des Bundesfinanzministeriums müssen im Jahr 2012 erstmals alle Bescheinigungen über die gemäß dem Gesetz zum Elterngeld und zur Elternzeit (BEEG) erhaltenen Leistungen an die Finanzverwaltung auf elektronischem Wege übersandt werden.

Durch die elektronische Übermittlung soll die bisherige, in Papierform erstellte „Jahresbescheinigung der Lohnersatzleistungen" ersetzt werden. Lediglich bei Unstimmigkeiten oder anderem berechtigten Interesse sollen Leistungsempfänger/-innen einen Ausdruck einer entsprechenden Bescheinigung oder von genaueren Informationen bzgl. der Datenübermittlung vom Träger der Sozialleistungen verlangen können.

IT.NRW betreibt und entwickelt zentral das Fachverfahren zum Elterngeld für die kreisfreien Städte und Kreise in Nordrhein-Westfalen. Nach Beauftragung durch die fachlich zuständige Bezirksregierung Münster konnte der Landesbetrieb zum 28.02.2012 für
293.000 Elterngeld-Antragsteller/-innen die Meldungen für erhaltene Leistungen bezogen auf das Jahr 2011 aus dem Fachverfahren ermitteln und an die Finanzverwaltung übersenden.

Die Datenübermittlung der Bescheinigungen von Lohnersatzleistungen erfolgte über die Schnittstelle zum Elster-Verfahren (Elster-Clearingstelle). Gleichzeitig musste IT.NRW über das Elterngeldverfahren zentral 293.000 elektronische Dokumente mit den an die Finanzverwaltung gemeldeten Daten erstellen und den elektronischen Elterngeldakten der jeweiligen Kommune (kreisfreie Städte und Kreise in NRW) zuordnen. Die Zuordnung der übermittelten Daten zu dem betreffenden Steuerpflichtigen erfolgte über die steuerliche Identifikationsnummer. Diese wird vom Bundeszentralamt für Steuern vergeben. Über das maschinelle Anfrageverfahren wurde bereits Ende 2011 für 98,7 Prozent der Elterngeld-Antragsteller/-innen die Steuer-ID ermittelt.

\section{BMWI-Studie: Standards bei Cloud Computing}

Der Parlamentarische Staatssekretär beim Bundesminister für Wirtschaft und Technologie, Hans-Joachim Otto, hat zur CeBIT 2012 eine aktuelle Studie zu Normung und Standardisierung beim Cloud Computing vorgestellt. Sie wurde vom Bundesminsterium für Wirtschaft und Technologie (BMWi) in Auftrag gegeben und von Booz \& Company sowie dem Forschungszentrum Informatik (FZI) erstellt.

Um die Standardisierung des Cloud Computing auf nationaler und europäischer Ebene besser aufeinander abzustimmen und zu beschleunigen, hat das BMWi bereits erste Gespräche mit dem DIN und der EU geführt. Unterstützt werden diese Bemühungen durch eine Arbeitsgruppe zur Standardisierung bei Cloud Computing, in der auch viele kleine und mittlere Unternehmen mitarbeiten. Standards beim Cloud Computing tragen zu mehr Sicherheit im Netz bei. Diese steht auch bei vielen mittelständischen Unternehmen ganz oben auf der Agenda. Die Gefahren bei der Nutzung von Informations- und Kommunikationstechnologien sind den meisten Unternehmerinnen und Unternehmern bekannt. Bei der konkreten Umsetzung besteht jedoch Nachholbedarf.

Eine Kurzfassung der Studie ist auf der Website des BMWi zu finden.

\section{Acronis: 10 Tipps für einen Disaster-Recovery-Plan}

Wie man einen effizienten Notfallmanagement-Prozess aufsetzt und welche Bereiche im Unternehmen bei der Planung berücksichtigt werden sollten, hat Acronis jetzt in zehn Punkten zusammengefasst.

\section{Analyse der Geschäftsprozesse}

In einem ersten Schritt sollte der Geltungsbereich des Notfallmanagements festgelegt werden. Hier sind zunächst alle Geschäftsprozesse zu identifizieren, die für das Unternehmen von essentieller Bedeutung sind. Bei den dabei zugrunde liegenden IT-Systemen hat das Unternehmen einen klaren Vorteil, wenn nach einem Schadensereignis sämtliche Daten inklusive Betriebssystem, Applikationen und Anwenderdaten in möglichst kurzer Zeit wieder komplett herstellbar sind. 